\title{
Centralized pain and pain catastrophizing mediate the association between lifetime abuse history and self-reported pain medication side effects
}

\author{
Jennifer Pierce, ${ }^{1}$ Afton L Hassett, ${ }^{1}$ Jill R Schneiderhan, ${ }^{2}$ Jude Divers, ${ }^{1}$ \\ Chad M Brummett, ${ }^{1}$ Jenna Goesling ${ }^{1}$
}

${ }^{1}$ Anesthesiology, University of Michigan Medical School, Ann Arbor, Michigan, USA

${ }^{2}$ Family Medicine, University of Michigan Medical School, Ann Arbor, Michigan, USA

Correspondence to Dr Jennifer Pierce,

Anesthesiology, University of Michigan, Ann Arbor, Ml 48108, USA; jmboik@med.umich.edu

Received 5 November 2019 Revised 6 January 2020 Accepted 10 January 2020 Published Online First 26 January 2020

\section{ABSTRACT \\ Background Self-reported side effects of pain medication are important determinants of treatment course that can affect patient adherence, medication discontinuation and physician decisions. Yet, few studies have investigated patient-level predictors of self-reported pain medication side effects. The present study sought to fill this gap by exploring the impact of physical or sexual abuse history on self-reported pain medication side effects and considered a mediation model in which those effects are transmitted through a centralized pain phenotype and pain catastrophizing. \\ Methods We conducted a cross-sectional analysis of 3118 patients presenting to a tertiary-care, outpatient pain clinic.}

Results Approximately $15 \%$ of the sample $(n=479)$ reported a lifetime history of abuse. Patients with a lifetime history of abuse, particularly abuse that occurred in both childhood and adulthood, reported more pain medication side effects compared with patients reporting no abuse history. Furthermore, path analysis showed that a centralized pain phenotype and pain catastrophizing mediated the association between lifetime abuse history and the sum of pain medication side effects.

Conclusions This suggests that individuals who experience abuse may develop a heightened physiological sensitivity to stimuli, as well as a tendency to interpret stimuli negatively, exaggerate the impact of aversive stimuli and undermine their ability to cope with the stressor. This study highlights the need for physicians to understand patient-level predictors of medication tolerance and to consider a history of abuse and trauma in decisions regarding treatment and medication management.

\section{INTRODUCTION}

All medications come with potential risks and side effects. Self-reported side effects of pain medication are important determinants of treatment course as they affect patient adherence, medication discontinuation and physician decisions. ${ }^{1}$ Somewhat surprisingly, patient-level predictors of self-reported side effects of pain medication have seldom been investigated. $^{2}{ }^{3}$ The present study explores the impact of abuse history on self-reported pain medication side effects. To our knowledge, only one previous study has linked abuse history with pain medication side effects. Specifically, Sansone, Sinclair and Wiederman found that the number of different types of childhood maltreatment experiences was positively correlated with self-reported medication allergies among patients with chronic pain. ${ }^{4}$ It is unclear, however, how and why abuse impacts pain medication side effects, which may be important for treatment decisions and patient-physician communication.

The present study considered two potential mediators of the association between abuse history and pain medication side effects: centralized pain and pain catastrophizing. Centralized pain, in which central nervous system factors contribute to the experience of pain and its perceptual amplification or spatial spread, has been linked to sensitivity to all sensory input, including both painful and non-painful stimuli (eg, odors, bright light). ${ }^{5}$ Previous research suggests that this centralized pain phenotype is exhibited to varying extents across a range of pain conditions and is associated with poorer functioning and pain-related outcomes. ${ }^{6}$ As such, it is frequently assessed on a continuum across diagnoses using the fibromyalgia survey criteria score. ${ }^{78}$ Although little is known about the potential link between centralized pain and pain medication side effects, heightened sensory sensitivity associated with centralized pain may translate to heightened physical sensitivity to medication. Alternatively, pain catastrophizing is a maladaptive cognitive and emotional reaction to pain in which individuals display an exaggerated negative cognitive and emotional response. ${ }^{9}$ This maladaptive response may translate to other aspects of pain management, including physical sensations related to and side effects from pain medication. Previous research suggests that catastrophizing is associated with higher likelihood of medication discontinuation and lower likelihood of pain relief from medication. ${ }^{10}$ Importantly, pain catastrophizing may impact the evaluation of physical effects of medication rather than the physical effects per se. ${ }^{11}$

Abuse history is associated with both indicators of centralized pain and pain catastrophizing. A history of abuse is associated with survey and experimental indicators of centralized pain, including the fibromyalgia survey criteria score, widespread pain and altered processing of controlled pain stimuli. $^{12} 13$ Abuse history is also associated with maladaptive coping and negative cognitive and emotional responses to future stressful stimuli. ${ }^{14}$ Cumulative abuse (herein defined as occurring in both childhood and adulthood) has been found to 


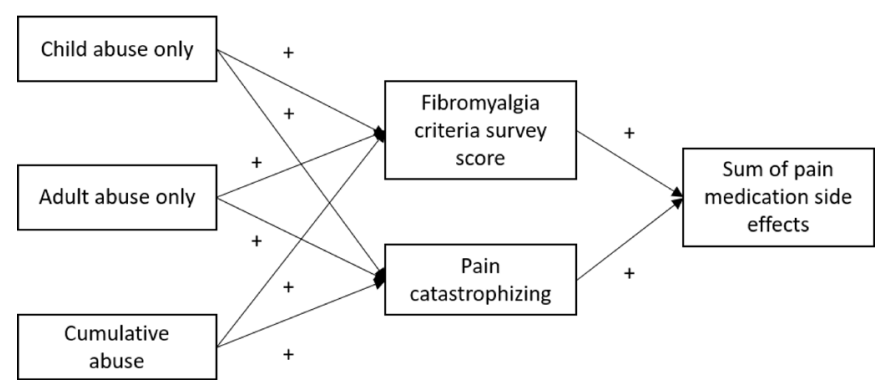

Figure 1 Hypothesized mediation model depicting the effect of lifetime abuse history on sum of pain medication side effects via fibromyalgia survey criteria score and pain catastrophizing.

be especially pernicious in its impact on health outcomes. ${ }^{15} 16$ The present study, therefore, explored the association between lifetime abuse history and pain medication side effects as mediated through centralized pain (ie, fibromyalgia survey criteria score) and pain catastrophizing (see figure 1 for the hypothesized model).

\section{METHODS}

\section{Participants and procedure}

The present study included patients 18 years of age and older who presented for care at a tertiary-care, outpatient pain clinic (Back \& Pain Center, Department of Anesthesiology, University of Michigan) between January 2011 and April 2016. All new patients completed a packet of questionnaires prior to their initial visit. The questionnaires are used for clinical care and research purposes. ${ }^{17}$ Previous studies have used the new patient data obtained through the University of Michigan Back \& Pain Center; however, our specific sample and research question differ from previous studies. ${ }^{13} 16$

\section{Measures}

Demographics: age and gender were obtained as part of patients' medical records.

Current and past pain medication use: as part of the new patient questionnaire, patients completed an exhaustive checklist of pain medications and were instructed to indicate current or past use for each medication. Current or former pain medications could include: non-steroidal anti-inflammatory drugs (eg, Aleve/Naprosyn; Tylenol; aspirin); narcotics/opioids (eg, morphine (MSContin); Vicodin/Norco/Lorcet); neuromodulators (eg, Elavil/amitriptyline; Cymbalta/duloxetine); anticonvulsants/membrane stabilizers (eg, Lyrica/pregabalin; Dilantin); muscle relaxants (eg, Soma, Zanaflex) or other pain medications (eg, Lidoderm patch; capsaicin; Catapress patch/clonidine; Valium/diazepam; Klonopin/clonazepam).

Self-reported pain medication side effects: after indicating current or past pain medications, patients were asked: Have you experienced side effects from your pain medications? Patients could then check boxes next to the following side effects: no side effects; confusion; constipation; diarrhea; difficulty urinating; dizziness; drowsiness; fatigue; hallucinations; heart palpitations; itchiness; mental impairment; nausea or vomiting; other stomach/bowel upset; shortness of breath; swelling hands and/or feet and/or other. Responses to the 'other' category that could fit into one of the other predefined categories were recoded. Individual analyses are not reported for 'other' responses. However, the 'other' category was included in the sum score of side effects, which was used for mediation analyses.
Lifetime abuse history: patients were asked: Do you have a history of physical or sexual abuse? Response options included 1 (yes) or 0 (no). Patients who responded yes were asked to indicate when this occurred, including childhood ( $<13$ years), adolescence (13-18 years) and adulthood. For the present study, childhood abuse included abuse experiences during childhood or adolescence. Therefore, four categories were formed: no abuse; child abuse only (including child and/or adolescent abuse but no adult abuse); adult abuse only (including adult abuse but no child or adolescent abuse) and cumulative abuse (including child and/or adolescent abuse and adult abuse).

Fibromyalgia survey criteria score: the 2011 American College of Rheumatology survey criteria for fibromyalgia (fibromyalgia survey criteria score) was used to assess centralized pain. This measure includes the Michigan Body $\mathrm{Map}^{18}$ to assess widespread pain across 19 body areas (0-19), as well as the Symptom Severity Index (SSI) to assess comorbid symptomatology (ie, fatigue, trouble thinking or remembering and headache). The SSI ranges from 0 to $12 .{ }^{8} 1819$ These scores are combined to create the FM survey score, ranging from 0 to 31 .

Pain catastrophizing: pain catastrophizing was measured using a subscale from the Coping Strategies Inventory. ${ }^{20}$ Patients were asked to consider six different statements related to how they think when they feel pain. Sample items include: "It's terrible, and I feel it's never going to get any better" and "I feel I can't stand it anymore". Response options range from 0 (never think that) to 6 (always think that). Scores are summed, and range from 0 to 36 .

\section{Data analysis plan}

Only patients who reported using some type of pain medication currently or in the past were included. Patients were also excluded if they did not provide information regarding abuse victimization history or had completely missing data for pain medication side effects (ie, did not indicate 'none' when no side effects were endorsed). Descriptive statistics were computed for the prevalence of side effects and lifetime abuse history, as well as scores on the fibromyalgia survey criteria and pain catastrophizing. Group differences in the prevalence of individual side effects by lifetime abuse history group were assessed using contingency analyses with associated $\chi^{2}$ tests. Significance was set at $\mathrm{p}<0.003$ to account for multiple comparisons.

Fibromyalgia survey criteria scores, pain catastrophizing and the sum of pain medication side effects were compared according to lifetime abuse history. Analysis of variance and Bonferroni post hoc comparisons were reported for fibromyalgia survey criteria score and pain catastrophizing comparisons. Welch's F test was used to determine omnibus differences in the sum of pain medication side effects due to violation of the homogeneity of variance assumption. Post hoc Games and Howell comparisons using 95\% CIs were used to determine specific group differences.

Path analysis was conducted. Age and gender were included in the model as covariates. Child abuse only, adult abuse only and cumulative abuse were included as distal binary predictors. Thus, no abuse was included as the reference category and direct and indirect effects are relative to this reference group. Full information maximum likelihood method of estimation was used to account for missing data. Bootstrap SEs were calculated with 500 samples. Overall model fit was evaluated with the $\chi^{2}$ goodness-of-fit test, the root mean square error of approximation (RMSEA), the comparative fit index (CFI) and the TuckerLewis index (TLI). RMSEA $\leq 0.05$ indicates good fit. CFI and 
Table 1 Differences in self-reported side effects from pain medication by lifetime abuse history

\begin{tabular}{|c|c|c|c|c|c|c|}
\hline & Total & No abuse & Child abuse only & Adult abuse only & Cumulative abuse & \\
\hline & $(n=3118)$ & $(n=2639)$ & $(n=320)$ & $(n=86)$ & $(n=73)$ & \\
\hline Side effect & $\%(n)$ & $\%(n)$ & $\%(n)$ & $\%(n)$ & $\%(n)$ & $P$ value \\
\hline Any side effect & $69.18(2157)$ & $67.56(1783)$ & $78.13(250)$ & $72.09(62)$ & $84.93(62)$ & $<0.001$ \\
\hline Drowsiness & 37.81 (1179) & $36.42(961)$ & $42.19(135)$ & $47.67(41)$ & $57.53(42)$ & $<0.001$ \\
\hline Constipation & $33.26(1037)$ & $31.68(836)$ & $39.69(127)$ & $41.86(36)$ & $52.05(38)$ & $<0.001$ \\
\hline Fatigue & $28.90(901)$ & $27.40(723)$ & $33.75(108)$ & $32.56(28)$ & $57.53(42)$ & $<0.001$ \\
\hline Dizziness & $22.71(708)$ & $21.94(579)$ & $25.00(80)$ & $24.42(21)$ & $38.36(28)$ & 0.007 \\
\hline Nausea or vomiting & $15.11(471)$ & $13.98(369)$ & $20.00(64)$ & $20.93(18)$ & $27.40(20)$ & $<0.001$ \\
\hline Confusion & $13.34(416)$ & $12.47(329)$ & $15.31(49)$ & $12.79(11)$ & 36.99 (27) & $<0.001$ \\
\hline Itchiness & $12.99(405)$ & $12.09(319)$ & $17.50(56)$ & $15.12(13)$ & $23.29(17)$ & 0.002 \\
\hline Other stomach/bowel upset & $11.26(351)$ & $10.19(269)$ & $17.19(55)$ & $12.79(11)$ & $21.92(16)$ & $<0.001$ \\
\hline Mental impairment & $8.50(265)$ & $7.77(205)$ & $10.94(35)$ & $10.47(9)$ & $21.92(16)$ & $<0.001$ \\
\hline Diarrhea & $7.02(219)$ & $6.14(162)$ & $10.00(32)$ & $11.63(10)$ & $20.55(15)$ & $<0.001$ \\
\hline Swelling hands and/or feet & $6.77(211)$ & $6.40(169)$ & $7.81(25)$ & $6.98(6)$ & $15.07(11)$ & 0.028 \\
\hline Difficulty urinating & $5.90(184)$ & $5.49(145)$ & $7.50(24)$ & $6.98(6)$ & $12.33(9)$ & 0.049 \\
\hline Heart palpitations & $5.26(164)$ & $4.70(124)$ & $6.25(20)$ & $6.98(6)$ & $19.18(14)$ & $<0.001$ \\
\hline Shortness of breath & $5.04(157)$ & $4.66(123)$ & $6.56(21)$ & $6.98(6)$ & $9.59(7)$ & 0.103 \\
\hline Hallucinations & $2.89(90)$ & $2.65(70)$ & $4.69(15)$ & $1.16(1)$ & $5.48(4)$ & 0.076 \\
\hline
\end{tabular}

Bold faced results are significant at Bonferonni-corrected $p<0.003$.

TLI $\geq 0.95$ indicates good fit. Bentler-Raykov squared multiple correlation coefficients $\left(\mathrm{R}^{2}\right)$ were assessed to determine the variance explained in the fibromyalgia survey criteria score, pain catastrophizing and sum of medication side effects due to the model. Specific indirect path coefficients were divided by the total indirect effect coefficient to determine the relative importance of each regressor and pathway. Analyses were conducted using StataIC V.15 (StataCorp, College Station, Texas, USA).

\section{RESULTS}

\section{Descriptive characteristics and group differences}

The present study included 3118 patients $\left(M_{\text {age }}=50.32\right.$, $\mathrm{SD}=15.38 ; 59.53 \%$ female). As seen in table 1 , nearly three out of four patients reported at least one side effect to pain medication. The five most common self-reported side effects to pain medications include: drowsiness (37.81\%), constipation (33.26\%), fatigue (28.90\%), dizziness $(22.71 \%)$ and nausea or vomiting (15.11\%). Abuse victimization was common, with $15.36 \%(n=479)$ reporting some form of physical or sexual abuse. Of those who experienced abuse, approximately $66.81 \%$ $(n=320)$ reported only child abuse victimization, $17.95 \%$ $(n=86)$ reported only adult abuse victimization, and $15.24 \%$ $(n=73)$ reported cumulative abuse victimization.

The prevalence of drowsiness, constipation, fatigue, nausea or vomiting, confusion, itchiness, other stomach/bowel upset, mental impairment, diarrhea and heart palpitations differed by abuse history (table 1). Across each type of side effect, patients who reported physical or sexual abuse more often reported each side effect compared with patients reporting no history of abuse. Victims of cumulative abuse reported the highest prevalence of side effects. Fibromyalgia survey criteria scores also differed according to lifetime abuse history, $F$ $(3, \mathrm{n}=2564)=60.85, \mathrm{p}<0.001$. As seen in figure 2 , patients reporting no abuse had lower fibromyalgia survey criteria scores compared with patients reporting only child abuse (Bonferroni adjusted $\mathrm{p}<0.001$ ), patients reporting only adult abuse (Bonferroni adjusted $\mathrm{p}<0.001)$ and patients reporting cumulative abuse (Bonferroni adjusted $\mathrm{p}<0.001$ ). Patients reporting cumulative abuse also had higher fibromyalgia survey criteria scores compared with patients reporting only child abuse (Bonferroni adjusted $\mathrm{p}<0.001$ ) and patients reporting only adult abuse (Bonferroni adjusted $\mathrm{p}=0.012$ ). Pain catastrophizing differed by lifetime abuse history, $F(3, \mathrm{n}=2924)=18.49, \mathrm{p}<0.001$. Patients reporting no abuse had lower pain catastrophizing scores compared with patients reporting child abuse only (Bonferroni adjusted $\mathrm{p}<0.001$ ), adult abuse only (Bonferroni adjusted $\mathrm{p}=0.032$ ) and cumulative abuse (Bonferroni adjusted $\mathrm{p}=0.006$ ). The sum of pain medication side effects reported also differed significantly by lifetime abuse history, Welch's $F$ (3, $181.41)=13.77, p<0.001$. As seen in figure 3 , patients reporting child abuse only had more symptoms compared with patients reporting no abuse (difference $=0.59, \mathrm{SE}=0.16,95 \% \mathrm{CI} 0.192$ to 0.993 ). Additionally, patients reporting cumulative abuse had more symptoms compared with patients reporting no abuse (difference $=2.16, \mathrm{SE}=0.42,95 \% \mathrm{CI} 1.055$ to 3.263 ), patients reporting child abuse only (difference $=1.57, \mathrm{SE}=0.44,95 \% \mathrm{CI}$ 0.407 to 2.726 and patients reporting adult abuse only (difference $=1.62, \mathrm{SE}=0.53,95 \% \mathrm{CI} 0.236$ to 3.005 ). The sum of pain medication side effects was also correlated with the fibromyalgia survey criteria score, $\mathrm{r}=0.30, \mathrm{p}<0.001$, and with pain catastrophizing, $\mathrm{r}=0.18, \mathrm{p}<0.001$. Additionally, fibromyalgia survey criteria scores were correlated with pain catastrophizing, $\mathrm{r}=0.39, \mathrm{p}<0.001$.

\section{PATH ANALYSIS}

Overall, the hypothesized model fit the data well, $\chi^{2}$ $(\mathrm{df}=3)=21.74, \quad \mathrm{p}<0.001 ; \quad \mathrm{RMSEA}=0.045 \quad(95 \%$ CI 0.028 to 0.063 ); $\mathrm{CFI}=0.981$; TLI $=0.888$. As seen in table 2 and figure 4 , reporting child abuse only, adult abuse only and cumulative abuse were all associated with higher fibromyalgia survey criteria scores and pain catastrophizing compared with reporting no abuse. In turn, higher fibromyalgia survey scores and higher pain catastrophizing were associated with more pain medication side effects. Younger age and female gender were associated with higher fibromyalgia survey criteria scores. Younger age was associated with higher pain catastrophizing and lower sum of pain medication side effects. 


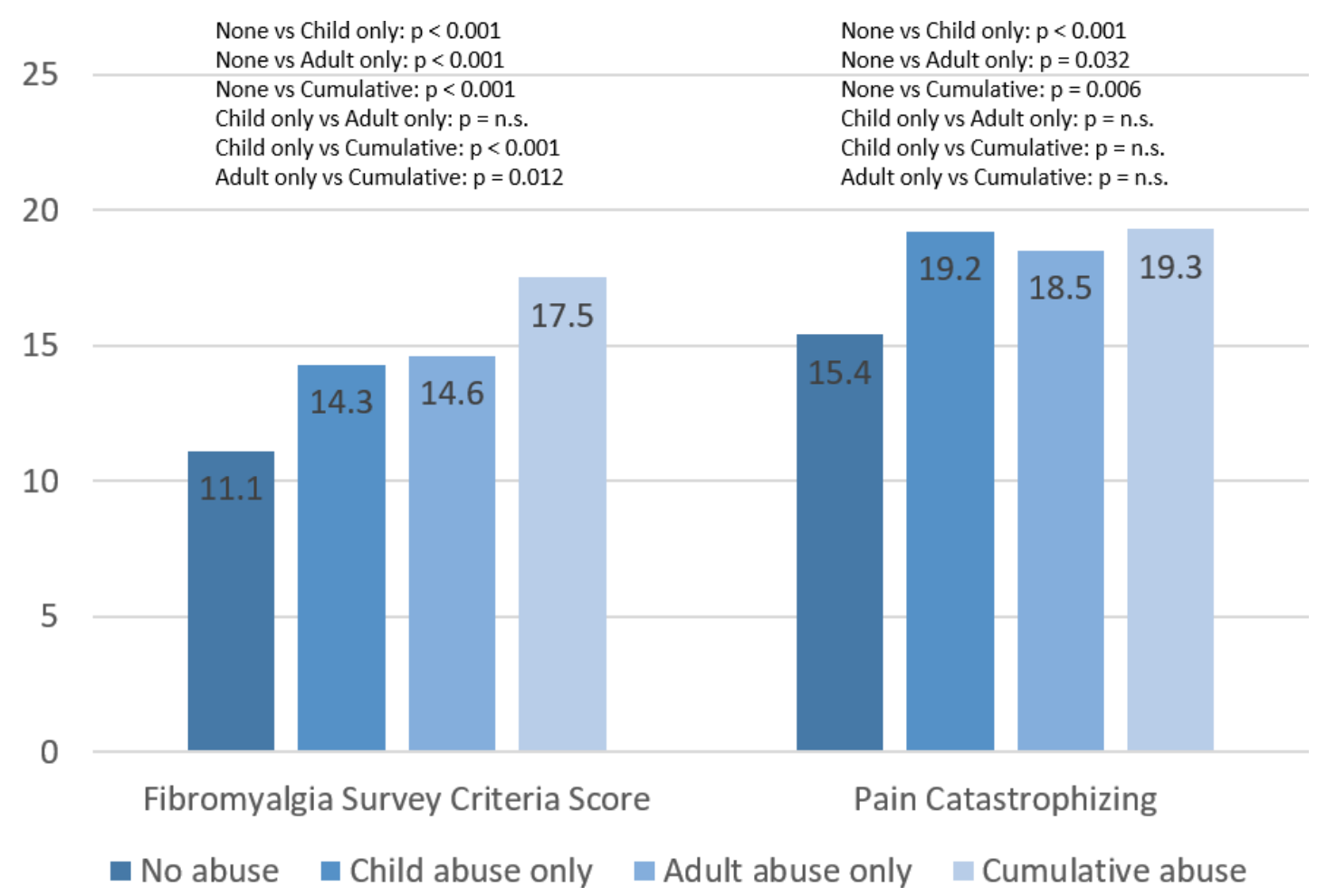

Figure 2 Average fibromyalgia survey criteria scores and pain catastrophizing according to lifetime abuse history. Omnibus comparisons were significant. Bonferroni post hoc comparisons are presented.

As seen in table 3 , each form of lifetime abuse history had an indirect effect on sum of pain medication side effects through the fibromyalgia survey criteria score and pain catastrophizing. Yet, when considering the proportion of the total indirect effect, cumulative abuse accounted for 48\%, whereas child abuse only accounted for $26 \%$ and adult abuse only accounted for $27 \%$. Furthermore, the path through fibromyalgia survey criteria scores accounted for more of the specific indirect effects across the forms of abuse, ranging from $81 \%$ to $90 \%$, compared with pain catastrophizing. The utility of the model was evaluated through the variance accounted for in the mediators and outcome. Eight per cent of the variance was accounted for in fibromyalgia survey criteria scores and $4 \%$ in pain catastrophizing. Furthermore, $10 \%$ of the variance was accounted for in the sum of pain medication side effects.

\section{DISCUSSION}

Although self-reported pain medication side effects can have a significant impact on treatment decisions, patient-level factors

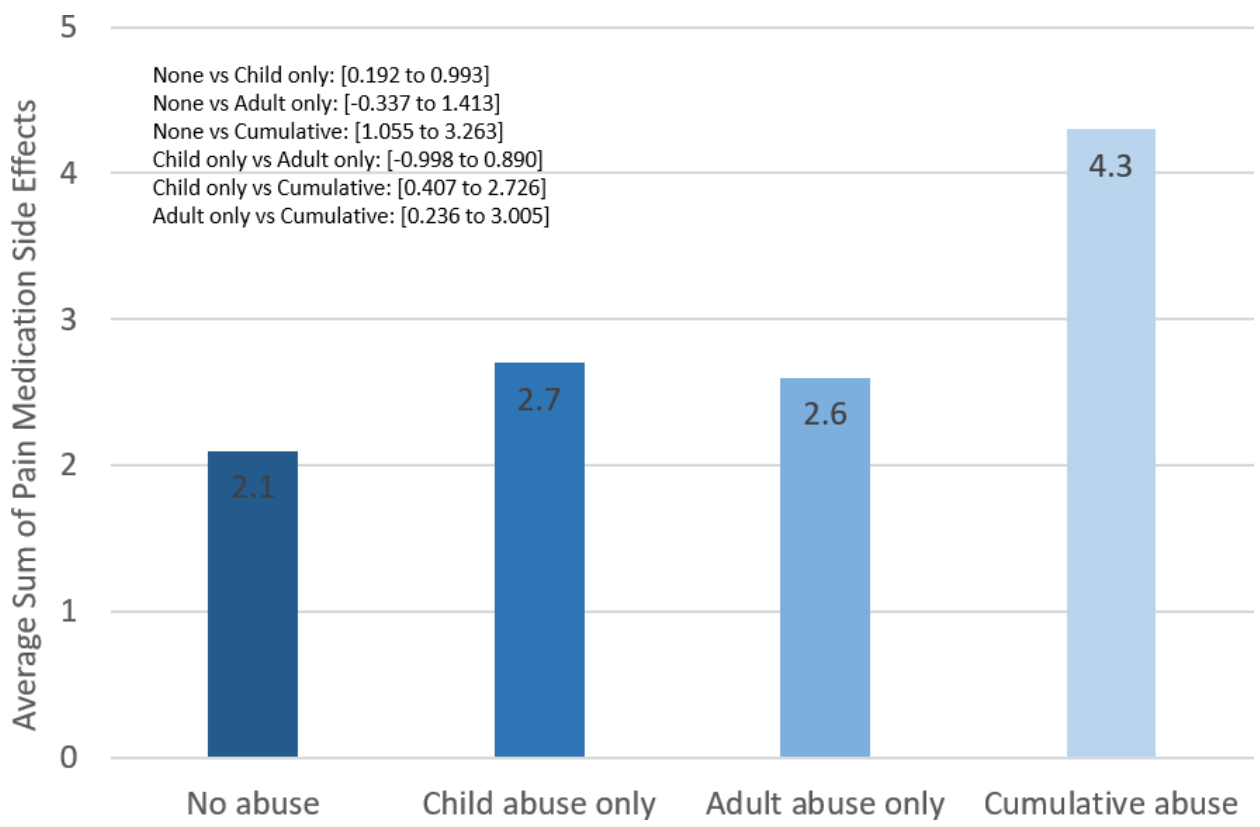

Figure 3 Average sum of pain medication side effects according to lifetime abuse history. Omnibus comparison was significant ( $<<0.001)$. Games and Howell $95 \%$ Cls are presented for post hoc comparisons. 
Table 2 Unstandardized coefficients, bootstrap SE and 95\% Cls for mediation model of the association between lifetime abuse history and sum of pain medication side effects $(n=3118)$

\begin{tabular}{|c|c|c|c|c|c|}
\hline & \multirow{2}{*}{$\begin{array}{l}\text { Unstandardized } \\
\text { coefficient }\end{array}$} & \multirow[b]{2}{*}{ Bootstrap SE } & \multirow[b]{2}{*}{$P$ value } & \multicolumn{2}{|l|}{$95 \% \mathrm{Cl}$} \\
\hline & & & & LL & UL \\
\hline \multicolumn{6}{|l|}{$\rightarrow \mathrm{FM}$ survey criteria score } \\
\hline Child abuse only & 2.92 & 0.37 & $<0.001$ & 2.198 & 3.647 \\
\hline Adult abuse only & 3.13 & 0.66 & $<0.001$ & 1.829 & 4.427 \\
\hline Cumulative abuse & 5.91 & 0.62 & $<0.001$ & 4.693 & 7.129 \\
\hline Age & -0.03 & 0.01 & $<0.001$ & -0.047 & -0.019 \\
\hline Female & 0.99 & 0.22 & $<0.001$ & 0.561 & 1.412 \\
\hline \multicolumn{6}{|l|}{$\rightarrow$ Pain catastrophizing } \\
\hline Child abuse only & 3.53 & 0.60 & $<0.001$ & 2.363 & 4.699 \\
\hline Adult abuse only & 2.94 & 1.08 & 0.006 & 0.823 & 5.055 \\
\hline Cumulative abuse & 3.66 & 0.99 & $<0.001$ & 1.733 & 5.596 \\
\hline Age & -0.10 & 0.01 & $<0.001$ & -0.122 & -0.076 \\
\hline Female & -0.28 & 0.36 & 0.440 & -0.988 & 0.430 \\
\hline \multicolumn{6}{|l|}{$\rightarrow$ Sum of pain medication side effects } \\
\hline FM survey criteria score & 0.12 & 0.01 & $<0.001$ & 0.100 & 0.140 \\
\hline Pain catastrophizing & 0.02 & 0.01 & $<0.001$ & 0.011 & 0.032 \\
\hline Age & 0.01 & 0.003 & 0.027 & 0.001 & 0.011 \\
\hline Female & 0.15 & 0.08 & 0.079 & -0.017 & 0.313 \\
\hline \multicolumn{6}{|l|}{ Covariate and lifetime abuse history covariance } \\
\hline FM survey criteria score $\leftrightarrow$ pain catastrophizing & 18.82 & 1.09 & $<0.001$ & 16.680 & 20.957 \\
\hline Child abuse only $\leftrightarrow$ adult abuse only & -0.003 & 0.0004 & $<0.001$ & -0.004 & -0.002 \\
\hline Child abuse only $\leftrightarrow$ cumulative abuse & -0.002 & 0.0003 & $<0.001$ & -0.003 & -0.002 \\
\hline Child abuse only↔age & -0.33 & 0.08 & $<0.001$ & -0.486 & -0.184 \\
\hline Child abuse only↔female & 0.02 & 0.003 & $<0.001$ & 0.016 & 0.026 \\
\hline Adult abuse only $\leftrightarrow$ cumulative abuse & -0.001 & 0.0001 & $<0.001$ & -0.001 & -0.0005 \\
\hline Adult abuse only $\leftrightarrow$ age & -0.05 & 0.04 & 0.133 & -0.126 & 0.017 \\
\hline Adult abuse only↔female & 0.01 & 0.001 & $<0.001$ & 0.007 & 0.012 \\
\hline Cumulative abuse $\leftrightarrow$ age & -0.09 & 0.03 & 0.003 & -0.153 & -0.031 \\
\hline Cumulative abuse $\leftrightarrow$ female & 0.01 & 0.001 & $<0.001$ & 0.007 & 0.011 \\
\hline Age $\leftrightarrow$ female & -0.21 & 0.14 & 0.122 & -0.486 & 0.057 \\
\hline
\end{tabular}

Bold faced results are significant at $\mathrm{p}<0.05$.

FM, fibromyalgia; LL, lower limit; UL, upper limit.

that predict side effects are seldom investigated. We sought to fill an important gap in knowledge by exploring how centralized pain and pain catastrophizing mediate the association between lifetime abuse history and pain medication side effects. Although the findings must be considered preliminary due to the limitations of our data, the findings may have important implications for the patient-physician relationship and treatment course.

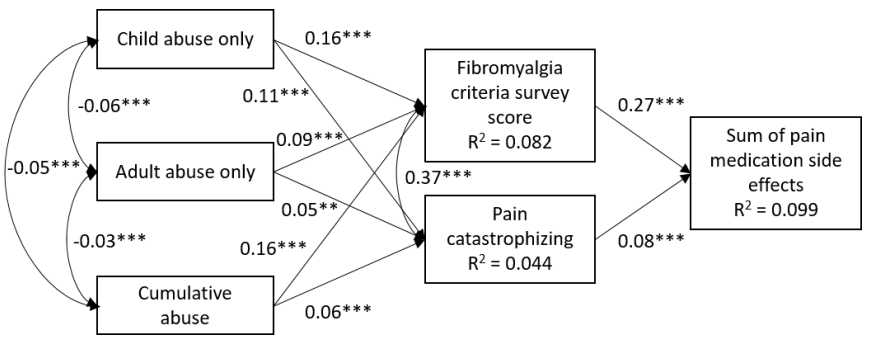

Figure 4 Mediation model of the effect of lifetime abuse history on sum of pain medication side effects via fibromyalgia survey criteria score and pain catastrophizing. Standardized coefficients are presented. Significance is determined using unstandardized coefficients and bootstrapped SEs. Solid lines indicate significant paths. Dashed lines indicate non-significant paths. ${ }^{*} \mathrm{P}<0.01,{ }^{* *} \mathrm{p}<0.001$. Covariates were included in estimation but are not shown. $\chi^{2}(n=3118)=21.74, p<0.001$. RMSEA $=0.045$ ( $95 \% \mathrm{Cl} 0.028$ to 0.063$)$. $\mathrm{CFI}=0.981, \mathrm{TLI}=0.888$.

Patients with a history of abuse, and cumulative abuse in particular, more often report pain medication side effects

Lifetime abuse history was common in our pain patient sample, with approximately $15 \%$ of our sample reporting a history of abuse. Previous research suggests that individuals with a history of trauma and abuse are more sensitive to internal and external stimuli, including differential responses to painful stimuli (eg, hyperalgesia) ${ }^{12}$; increased sensitivity to changes in sensory information ${ }^{21}$; increased self-reported sensitivity to sensory stimuli ${ }^{22}$ and a heightened sense that internal stimuli are noxious (eg, anxiety sensitivity). ${ }^{23}$ Our finding that patients with a lifetime history of abuse reported more medication side effects suggests that, in the context of chronic pain treatment, some patients experience adverse effects to the very same medications offered to provide relief potentially through heightened sensitivity. Furthermore, previous research suggests that victims of abuse use healthcare services more and report more pain medication use than individuals who had not experienced abuse. $^{24}$ This creates a paradox whereby individuals with a history of abuse display greater need and desire for pharmacological treatment, yet may be less able to tolerate it and attain benefits. This process makes it more difficult for physicians to treat patients with a history of trauma, and necessitates an understanding of such background factors impacting treatment decisions and responses. 
Table 3 Total and specific indirect effects, bootstrap SEs and 95\% Cls for mediation model of the association between lifetime abuse history and sum of pain medication side effects $(n=3118)$

\begin{tabular}{|c|c|c|c|c|c|c|c|}
\hline & \multirow{2}{*}{$\begin{array}{l}\text { Unstandardized } \\
\text { coefficient }\end{array}$} & \multirow{2}{*}{$\begin{array}{l}\text { Bootstrap } \\
\text { SE }\end{array}$} & \multirow[b]{2}{*}{$P$ value } & \multicolumn{2}{|l|}{$95 \% \mathrm{Cl}$} & \multirow{2}{*}{$\begin{array}{l}\text { Proportion of total } \\
\text { indirect effect }\end{array}$} & \multirow{2}{*}{$\begin{array}{l}\text { Proportion of specific } \\
\text { indirect effect }\end{array}$} \\
\hline & & & & LL & UL & & \\
\hline Total indirect effect & 1.66 & 0.19 & $<0.001$ & 1.286 & 2.028 & & \\
\hline Child abuse only $\rightarrow$ sum of pain medication side effects & 0.43 & 0.06 & $<0.001$ & 0.312 & 0.544 & 0.26 & \\
\hline$\rightarrow$ Fibromyalgia survey criteria score & 0.35 & 0.05 & $<0.001$ & 0.246 & 0.457 & & 0.81 \\
\hline$\rightarrow$ Pain catastrophizing & 0.08 & 0.02 & 0.001 & 0.030 & 0.123 & & 0.19 \\
\hline Adult abuse only $\rightarrow$ sum of pain medication side effects & 0.44 & 0.09 & $<0.001$ & 0.255 & 0.624 & 0.27 & \\
\hline$\rightarrow$ Fibromyalgia survey criteria score & 0.38 & 0.09 & $<0.001$ & 0.207 & 0.544 & & 0.86 \\
\hline$\rightarrow$ Pain catastrophizing & 0.06 & 0.03 & 0.020 & 0.010 & 0.118 & & 0.14 \\
\hline Cumulative abuse $\rightarrow$ sum of pain medication side effects & 0.79 & 0.10 & $<0.001$ & 0.585 & 0.994 & 0.48 & \\
\hline$\rightarrow$ Fibromyalgia survey criteria score & 0.71 & 0.10 & $<0.001$ & 0.514 & 0.906 & & 0.90 \\
\hline$\rightarrow$ Pain catastrophizing & 0.08 & 0.03 & 0.008 & 0.021 & 0.138 & & 0.10 \\
\hline Age & -0.01 & 0.001 & $<0.001$ & -0.008 & -0.004 & & \\
\hline Female & 0.11 & 0.03 & $<0.001$ & 0.050 & 0.174 & & \\
\hline
\end{tabular}

Total indirect effect refers to effect from predictors of interest (not including age and female).

LL, lower limit; UL, upper limit.

An increased reporting of pain medication side effects may be an additional consequence to add to the sequelae of abuse. ${ }^{4}$ A history of abuse is associated with a more complex symptom presentation and worse functioning in multiple domains, including worse psychological health, more widespread pain, higher pain interference and worse functioning. ${ }^{13}$ Abuse in childhood and adulthood is also associated with increased reporting of somatic symptoms. ${ }^{25} 26$ In the present study, patients experiencing abuse in both childhood and adulthood had the highest frequency of reporting medication side effects. Previous research supports the finding that cumulative abuse is particularly impactful. Polyvictimization and the sum of different types of maltreatment, as well as abuse across the lifespan (ie, cumulative abuse as defined in the present study), are associated with more severe health consequences. ${ }^{15} 2527$ The present study suggests that this extends to the self-reported adverse effects of pain medication.

\section{The effect of abuse on self-reported pain medication side effects is mediated through a centralized pain phenotype and pain catastrophizing}

Our findings showed that patients with a lifetime history of abuse reported higher scores on fibromyalgia survey criteria, pain catastrophizing and the sum of pain medication side effects. Path analyses further revealed that fibromyalgia survey criteria scores and pain catastrophizing mediated the association between lifetime history of abuse and the sum of pain medication side effects. Centralized pain may be a potent consequence of trauma and abuse that induces numerous negative health complaints. Indeed, a recent meta-analysis found that individuals reporting trauma (ie, post-traumatic stress disorder (PTSD), combat or abuse) were 2.7 times more likely to report a centralized pain syndrome, including fibromyalgia, chronic widespread pain, chronic fatigue syndrome, temporomandibular disorder and irritable bowel syndrome. ${ }^{28}$ Previous research suggests that a centralized pain phenotype mediates the association between abuse history and pain-related outcomes, including pain severity and physical function. ${ }^{13}$ Centralized pain is also associated with heightened sensitivity to a range of stimuli, including chemical sensitivity. ${ }^{29}$ Thus, trauma and abuse may render an individual more sensitive to stimuli, manifested by a centralized pain phenotype, and contribute to how a patient experiences bodily symptoms as they are taking a new medication that targets their central nervous system.

Interestingly, the impact of abuse on the prevalence of pain medication side effects was consistent across all reported symptoms. These symptoms mirror the somatic symptoms more often reported among victims of abuse. ${ }^{2526}$ Although it is unclear if adverse side effects of medication simply represent a misattribution of somatic symptoms due to the use of medication, the link between trauma and centralized pain suggests that there is physiological sensitivity. We were unable, however, to distinguish somatic complaints from true adverse pain medication side effects in the present study. Still, it is not clear that such a distinction matters clinically. Additionally, much of the research in non-specific medication side effects has explored the nocebo effect, which is defined as adverse reactions that are not directly attributable to the pharmacological action of the drug and are frequently attributed to negative expectations regarding the drug and other psychological factors. ${ }^{2}$ Yet, our findings related to the impact of a centralized pain phenotype on pain medication side effects suggests the potential for unique, patient-specific physiological responses to pain medication. Because a lifetime history of abuse is linked to the experience of centralized pain, our findings suggest that this may be a potent mechanism through which abuse victims display negative effects to pain medication.

The potential, however, for psychological mechanisms to convey non-specific, adverse responses to medication was affirmed by our finding that pain catastrophizing was also a significant mediator. This suggests that individuals who experience abuse may develop a tendency to interpret stimuli negatively, exaggerate the impact of aversive stimuli and undermine their ability to cope with the stressor. This is similar to previous work that has found an association between trauma and anxiety sensitivity, which is described as an exaggerated fear of anxiety, anxiety-related sensations and their consequences. ${ }^{23}$ Pain catastrophizing may also represent an attentional bias toward and inability to disengage from pain cues. ${ }^{9}$ Indeed, hypervigilance for threat is a hallmark symptom of PTSD. In turn, our findings suggest that the tendency to attend to and exaggerate negative pain-related stimuli may extend to the reporting of adverse pain medication side effects. Importantly, however, a centralized pain phenotype accounted for more of the indirect effect on the sum 
of pain medication side effects compared with pain catastrophizing. Therefore, although abuse may impact both the sensory perception and evaluation of medication-related effects, the sensory perception of physical reactions to the medication may be more important in patient reporting of side effects. ${ }^{11}$

\section{Limitations}

Several limitations are worth noting. The present study used data collected in the context of clinical care. Therefore, our measures were limited. We were unable to determine when medications were taken, as well as how many types and how much of each type of medication were consumed. The pain medication side effects were reported for any pain medication and could not be connected to any specific type of medication. Additionally, retrospective reports of symptoms from the distant past may elicit different memory processes compared with proximal events. For example, the former may be influenced more by beliefs about the self (ie, semantic memory) compared with recent events, which are autobiographical in nature. ${ }^{30}$ It is unclear how this bias may have influenced the current results. Future research should more carefully control for type, amount, time frame and diversity of pain medication treatment and associated side effects. We were also unable to determine how severe the participant would rate the side effect and whether this prompted medication discontinuation. Additionally, the reporting of side effects is subjective and was not verified by an external source. As previously indicated, research suggests that patient characteristics (eg, personality traits) predict nocebo effects, or negative side effects to inert substances. ${ }^{2}$ Somatic symptoms not caused by medication may also be misattributed to pain medication side effects. ${ }^{2}$ Thus, future research should consider these other possible explanations as potential mediators. The measure of abuse was also limited; future studies would benefit from incorporating the type, frequency and severity of abuse, as well as other forms of maltreatment (eg, emotional abuse). Furthermore, many victims of abuse do not acknowledge or label it as such. Because our measure of abuse did not provide behavioral descriptors of what the abuse would entail (ie, hitting, kicking), our measure may not have included all individuals who experienced physical or sexual abuse in their lifetime. Many factors may also bias abuse reporting using retrospective measures. For example, healthy individuals are less likely to retrospectively report documented abuse. ${ }^{31}$ The present sample included patients with a wide variety of pain-related symptoms and diagnoses. Future research may also consider how specific pain diagnoses (ie, fibromyalgia, complex regional pain syndrome, osteoarthritis) modify the model presented herein. Finally, cross-sectional studies cannot provide information about directional effects. Longitudinal and experimental studies are needed to elucidate causal pathways. For these reasons, the present study should be considered a preliminary exploration of the link between abuse and pain medication side effects that supports future research addressing these limitations.

\section{Clinical implications and conclusion}

In chronic pain conditions, adherence to a medication regimen can be particularly important in providing relief. Yet, adverse pain medication side effects negatively impact decisions regarding adherence. ${ }^{1}$ Patients may alter their medication use to minimize side effects. This study highlights the importance of physicians having a thorough understanding of patient-level predictors, particularly a history of trauma and abuse, prior to beginning a course of treatment and being willing to modify consultation, administration and monitoring practices with this knowledge in mind. Non-adherence due to negative side effects will not be altered unless the concern regarding negative side effects is properly and thoroughly addressed. ${ }^{32}$ Physicians might consider patient education regarding the benign and transitory nature of some side effects and consider lower starting doses, which may help prevent discontinuation of some of these medications in certain situations. Importantly, when patients with a history of abuse report hypersensitivity to medication and poor adherence, physicians should be aware of centralized pain states that may amplify negative medication side effects and consider a history of abuse and trauma in decisions regarding treatment and medication management.

\section{Twitter Chad M Brummett @drchadb}

Acknowledgements The authors would like to thank the patients who participated in the study, the research team, the staff of the Back and Pain Center and Dr Stephanie Moser for insightful discussions.

Contributors JP: responsible for conception and design of work; data management, analysis, presentation and interpretation; drafting the article; critical revision of the article and final approval of version to be published. $\mathrm{AH}, \mathrm{CMB}$ and JG: responsible for study design; interpretation of the data; critical revision of the manuscript and final approval of the version to be published. JRS and JD: responsible for critical revision of the manuscript and final approval of version to be published.

Funding This research was supported by the Department of Anesthesiology, University of Michigan. JP is supported by a Postdoctoral Translational Scholar Programme training grant from the Michigan Institute for Clinical and Health Research (UL1TR002240; Pl: George Mashour).

Competing interests ALH is a consultant to Precision Health Economics and AbbVie Pharmaceuticals. CMB is a consultant for Heron Therapeutics.

Patient consent for publication Not required.

Ethics approval Institutional Review Board (University of Michigan, Ann Arbor, Michigan, USA) approval was obtained and informed consent was waived.

Provenance and peer review Not commissioned; externally peer reviewed.

Data availability statement Deidentified participant data are available on reasonable request from the authors.

\section{REFERENCES}

1 Timmerman L, Stronks DL, Groeneweg JG, et al. Prevalence and determinants of medication non-adherence in chronic pain patients: a systematic review. Acta Anaesthesiol Scand 2016;60:416-31.

2 Barsky AJ, Saintfort R, Rogers MP, et al. Nonspecific medication side effects and the nocebo phenomenon. JAMA 2002;287:622-7.

3 Barsky AJ, Orav EJ, Ahern DK, et al. Somatic style and symptom reporting in rheumatoid arthritis. Psychosomatics 1999;40:396-403.

4 Sansone R, Sinclair J, Wiederman M. Drug allergies and childhood trauma among chronic pain patients. Psychiatry 2009;6:17-18.

5 Harte SE, Harris RE, Clauw DJ. The neurobiology of central sensitization. J App/ Biobehav Res 2018:23:e12137.

6 Brummett CM, Janda AM, Schueller CM, et al. Survey criteria for fibromyalgia independently predict increased postoperative opioid consumption after lowerextremity joint arthroplasty: a prospective, observational cohort study. Anesthesiology 2013:119:1434-43.

7 Clauw DJ. Fibromyalgia: a clinical review. JAMA 2014;311:1547-55.

8 Wolfe F, Clauw DJ, Fitzcharles M-A, et al. Fibromyalgia criteria and severity scales for clinical and epidemiological studies: a modification of the ACR preliminary diagnostic criteria for fibromyalgia. J Rheumatol 2011;38:1113-22.

9 Quartana PJ, Campbell CM, Edwards RR. Pain catastrophizing: a critical review. Expert Rev Neurother 2009:9:745-58.

10 Toth C, Brady S, Hatfield M. The importance of catastrophizing for successful pharmacological treatment of peripheral neuropathic pain. J Pain Res 2014;7:327-38

11 Geisser ME, Robinson ME, Keefe FJ, et al. Catastrophizing, depression and the sensory, affective and evaluative aspects of chronic pain. Pain 1994;59:79-83.

12 Tesarz J, Gerhardt A, Leisner S, et al. Distinct quantitative sensory testing profiles in nonspecific chronic back pain subjects with and without psychological trauma. Pain 2015:156:577-86.

13 Nicol AL, Sieberg CB, Clauw DJ, et al. The association between a history of lifetime traumatic events and pain severity, physical function, and affective distress in patients with chronic pain. J Pain 2016;17:1334-48. 
14 Valle LA, Silovsky JF. Attributions and adjustment following child sexual and physical abuse. Child Maltreat 2002;7:9-24.

15 Follette VM, Polusny MA, Bechtle $A E$, et al. Cumulative trauma: the impact of child sexual abuse, adult sexual assault, and spouse abuse. J Trauma Stress 1996;9:25-35

16 Pierce J, Moser S, Hassett AL, et al. Influence of abuse history on concurrent benzodiazepine and opioid use in chronic pain patients. J Pain 2019;20:473-80.

17 Hassett AL, Wasserman R, Goesling J, et al. Longitudinal assessment of pain outcomes in the clinical setting: development of the "APOLO" electronic data capture system. Reg Anesth Pain Med 2012;37:398-402.

18 Brummett CM, Bakshi RR, Goesling J, et al. Preliminary validation of the Michigan body MAP. Pain 2016;157:1205-12.

19 Wolfe F, Clauw DJ, Fitzcharles M-A, et al. The American College of rheumatology preliminary diagnostic criteria for fibromyalgia and measurement of symptom severity. Arthritis Care Res 2010;62:600-10.

20 Swartzman LC, Gwadry FG, Shapiro AP, et al. The factor structure of the coping strategies questionnaire. Pain 1994;57:311-6.

21 Morgan CA, Grillon C. Abnormal mismatch negativity in women with sexual assaultrelated posttraumatic stress disorder. Biol Psychiatry 1999;45:827-32.

22 Serafini G, Gonda X, Pompili M, et al. The relationship between sensory processing patterns, alexithymia, traumatic childhood experiences, and quality of life among patients with unipolar and bipolar disorders. Child Abuse Negl 2016;62:39-50.

23 Marshall GN, Miles JNV, Stewart SH. Anxiety sensitivity and PTSD symptom severity are reciprocally related: evidence from a longitudinal study of physical trauma survivors. J Abnorm Psychol 2010;119:143-50.
24 Finestone HM, Stenn P, Davies F, et al. Chronic pain and health care utilization in women with a history of childhood sexual abuse. Child Abuse Negl 2000:24:547-56

25 Walker EA, Gelfand A, Katon WJ, et al. Adult health status of women with histories of childhood abuse and neglect. Am J Med 1999;107:332-9.

26 Sansone RA, Gaither GA, Sansone LA. Childhood trauma and adult somatic preoccupation by body area among women in an internal medicine setting: a pilot study. Int J Psychiatry Med 2001;31:147-54.

27 Felitti VJ, Anda RF, Nordenberg D, et al. Relationship of childhood abuse and household dysfunction to many of the leading causes of death in adults. The adverse childhood experiences (ACE) study. Am J Prev Med 1998;14:245-58.

28 Afari N, Ahumada SM, Wright LJ, et al. Psychological trauma and functional somatic syndromes: a systematic review and meta-analysis. Psychosom Med 2014;76:2-11.

29 Clauw DJ, Chrousos GP. Chronic pain and fatigue syndromes: overlapping clinical and neuroendocrine features and potential pathogenic mechanisms. Neuroimmunomodulation 1997:4:134-53.

30 Van den Bergh 0, Walentynowicz M. Accuracy and bias in retrospective symptom reporting. Curr Opin Psychiatry 2016;29:302-8.

31 Hardt J, Rutter M. Validity of adult retrospective reports of adverse childhood experiences: review of the evidence. J Child Psychol Psychiatry 2004;45:260-73.

32 Garner JB. Problems of nonadherence in cardiology and proposals to improve outcomes. Am J Cardiol 2010;105:1495-501. 